\title{
Control of Plasma Cholesterol-Lowering Action of Probucol with Various Lipid Carrier Systems
}

\author{
Toichi Takino, ${ }^{a}$ Noriko Koreeda, ${ }^{a}$ Takehiko Nomura, ${ }^{a}$ Toshiyuki Sakaeda (né Kakutani), ${ }^{b}$ \\ Fumiyoshi Yamashita, ${ }^{a}$ Yoshinobu TAKakura, ${ }^{a}$ and Mitsuru Hashida ${ }^{*, a}$ \\ Department of Drug Delivery Research, Graduate School of Pharmaceutical Sciences, Kyoto University, ${ }^{a}$ Yoshida \\ Shimoadachi-cho, Sakyo-ku, Kyoto 606-8501, Japan and Shionogi Research Laboratories, Shionogi and Co., Ltd., \\ Fukushima-ku, Osaka 553-0002, Japan. Received October 23, 1997; accepted February 13, 1998
}

\begin{abstract}
In order to explore the relationship between the pharmacokinetic properties and pharmacological actions of lipophilic drugs injected with lipid carrier systems, probucol was selected as a model drug with high lipophilicity, and the effect of disposition control on cholesterol-lowering activities was evaluated. Both large emulsion, with mean diameter of $280 \mathrm{~nm}$, and long-circulating type small emulsion containing egg sphingomyelin with mean diameter of $100 \mathrm{~nm}$, showed stable incorporation of probucol. The former produced rapid accumulation of probucol in the liver, while the latter demonstrated prolonged systemic circulation and gradual hepatic uptake. On the other hand, injection of a micellar solution with HCO-60 (polyoxyethylene hydrogenated castor oil) showed a rapid decrease in plasma concentration and a high hepatic uptake of probucol, similar to injections with serum, suggesting the rapid release of the drug from the micelles. However, probucol in a micellar solution showed higher cholesterol-lowering action than that in emulsion formulations. These results suggested that the pharmacological action of probucol in the liver might be affected by the uptake mode and sequential disposition in the organ, depending on the drug retention properties of the lipid carrier particles.
\end{abstract}

Key words probucol; biodistribution control; cholesterol-lowering action; lipid carrier system; polyoxyethylene hydrogenated castor oil (HCO-60) micelle; o/w emulsion

Probucol is a hypo-cholesterolemic agent which exerts a marked influence on plasma lipoprotein and apolipoprotein concentration by selectively influencing the apo A-IV and low density lipoprotein (LDL)-receptor gene expression in hepatocytes. It is usually administered by an oral route at an extremely high dose in clinical usage due to its very low water solubility and subsequent poor gastrointestinal absorption (less than $10 \%) .{ }^{1)}$ This is a fairly general problem with highly lipophilic drugs, so that the development of an effective administration method for these drugs has attracted great interest in the pharmaceutical sciences. Parenteral administration with lipid carrier systems is considered to be one feasible approach for solving this problem as well as for controlling in vivo disposition of highly lipophilic drugs. Particulate lipid carriers such as liposomes, o/w emulsion, and micelles have been developed as drug carrier systems and have been clinically applied. ${ }^{2-8)}$ However, the pharmacokinetic behavior of highly lipophilic drugs injected with lipid carriers which might be affected by the drug/carrier interaction has not been studied extensively. ${ }^{9)}$

In our previous reports, the feasibility of lipid carrier systems such as liposomes, o/w emulsion and micelles were studied. The factors governing their delivery potentials were clarified by in vivo biodistribution studies ${ }^{10,11)}$ and by singlepass rat liver perfusion experiments. ${ }^{12)}$ In these studies, lipid carrier systems were shown to exhibit their potentials via stable drug incorporation which arose from conditions such as the sufficient lipophilicity of the objective drugs and the retentive ability of carriers based on their physicochemical structure and lipid composition. That is, the necessary lipophilicity of the drugs was clarified to be higher than $10^{6}$ for liposomes, $10^{9}$ for emulsions and $10^{12}$ for non-ionic surfactant $\mathrm{HCO}-60$ (polyoxyethylene hydrogenated castor oil) micelles on the basis of the partition coefficient between $n$ octanol and water $\left(\mathrm{PC}_{\mathrm{oct}}\right)$. Lipophilic drugs satisfying these

* To whom correspondence should be addressed. criteria were shown to be delivered with lipid carriers after intravenous administration, while drugs with low lipophilicity demonstrated their original disposition profiles after being released from the lipid carriers. However, little information has been obtained about the effect of the disposition control on the pharmacological potency of lipophilic drugs.

Under these situations, probucol with a $\mathrm{PC}_{\text {oct }}$ of $10^{10.8}$ was selected as a model drug which showed diverse distribution profiles with or without being incorporated into the carriers, and its pharmacological effect is evaluated in relation to its biodistribution profiles.

\section{MATERIALS AND METHODS}

Chemicals [propane- $\left.2-{ }^{14} \mathrm{C}\right]$ Probucol $\quad(370 \mathrm{MBq} / \mathrm{mmol})$ was purchased from Amersham International plc, England. [Cholesteryl-1, 2- ${ }^{3} \mathrm{H}$ ]cholesteryl hexadecyl ether $(2.00 \mathrm{GBq} /$ mmol) was purchased from Daiichi Radioisotopes, Tokyo, Japan. Probucol and egg sphingomyelin (SM) were purchased from Sigma Chemical Co., U.S.A. Intralipid $\left.{ }^{(}\right)(10 \%)$ was purchased from Otsuka Pharmaceutical Co., Tokushima, Japan. Egg phosphatidylcholine (PC) was purchased from Nippon Oil and Fats Co., Hyogo, Japan. Soybean oil was purchased from Wako Pure Chemicals Industries, Ltd., Osaka, Japan. All other chemicals were of the finest grade available.

Estimation of Lipophilicities Lipophilicity was calculated in terms of the $\mathrm{PC}_{\text {oct }}$ by Hansch and Leo's fragment method using a computer program CLOGP3 in MedCem Software Release 3.54 Programs 1989, Chemical Information System, Inc., Claremont, CA, U.S.A. ${ }^{13,14)}$

Preparation of Injection Formulations for in Vivo Distribution Study The lipid carrier systems tested were prepared in the same way as those employed in our series of pharmacokinetic studies for model lipophilic compounds. ${ }^{10,11)}$ A large emulsion was prepared by diluting Intralipid ${ }^{\circledR}(10 \%)$ 
with the same volume of water, and its lipid composition is $P C$ : soybean oil $=0.12: 1$. Small emulsion was prepared with PC, SM and soybean oil $(0.7: 0.3: 0.1)$ by sonication (Otake Works, Output $200 \mathrm{~W}, 60 \mathrm{~min})$. Liposomes were prepared from PC and SM ( $7: 3)$ by a combination of controlled dialysis using a Lipoprep ${ }^{\circledR}$ dialyzer (Diachema) and sequential extrusion. ${ }^{15)}$ In all the formulations, including the HCO-60 micellar solution, the final lipid content was adjusted to $5 \%$.

After evaporation of a mixed solvent of toluene and ethanol containing $\left[{ }^{3} \mathrm{H}\right]$ cholesteryl hexadecyl ether $(0.74$ $\mathrm{MBq}),\left[{ }^{14} \mathrm{C}\right]$ probucol $(0.074 \mathrm{MBq})$ and probucol $(22 \mu \mathrm{g})$ under reduced pressure, $8 \mathrm{ml}$ of large or small emulsion, liposome, HCO-60 micellar solution, or mouse serum was added, and the precipitate was dissolved by overnight incubation. Each preparation was filtered through a Millex HV sterile filter (Millipore) before the animal experiments. The particle size of the lipid carrier systems was measured by a laser particle analyzer (Photal SL-900 (Dynamic Light Scattering Spectrophotometer DLS-700, Laser Particle Analyzer System LPA-3100), Otsuka Electronics Co., Osaka).

In Vivo Distribution Experiment Male ddY mice $(25-28 \mathrm{~g})$ were obtained from the Shizuoka Agricultural Co-operative Association for Laboratory Animals, Shizuoka. Lipid carrier systems containing $\left[{ }^{3} \mathrm{H}\right]$ cholesteryl hexadecyl ether, $\left[{ }^{14} \mathrm{C}\right]$ probucol and probucol $(22 \mu \mathrm{g})$ were injected into the tail vein on mice at a volume of $5 \mathrm{ml} / \mathrm{kg}$. At appropriate intervals after injection, blood was collected from the vena cava under ether anesthesia and the mice were sacrificed. The lung, liver, and spleen were excised, rinsed with saline, weighed, and subjected to assay.

The radioactivities of the blood and tissue were measured using a liquid scintillation counter (LSC-5000, Beckman) after the blood and tissue were dissolved with Soluene-350 (Packard, Netherlands), decolorized by hydration peroxide, and to which scintillation medium Clear-sol I (Nacalai Tesque, Kyoto) was added. Contamination of the blood in the tissue samples was corrected using the distribution data of $\left[{ }^{14} \mathrm{C}\right]$ carboxymethyl-dextran (T-70) at $1 \mathrm{~h}$ after intravenous injection. ${ }^{16)}$

Pharmacokinetic Analysis Tissue distribution data were evaluated in terms of the tissue uptake rate index as reported previously. ${ }^{10,11)}$ Changes in the amount of radioactivity in a tissue, over time, can be described as follows:

$$
\frac{d T(t)}{d t}=C l_{\text {in }} \times C(t)-K_{\text {out }} \times T(t)
$$

where $T(\%$ of dose $/ \mathrm{g})$ is the amount of radioactivity in $1 \mathrm{~g}$ of the tissue, $C(\%$ of dose $/ \mathrm{ml})$ is the blood concentration of radioactivity, $C l_{\text {in }}(\mathrm{ml} / \mathrm{h} / \mathrm{g})$ is the tissue uptake rate index (clearance) from the blood to the tissue, and $K_{\text {out }}(1 / \mathrm{h})$ is the efflux rate constant from the tissue. To estimate the uptake rate index, distribution in the early phase ( $30 \mathrm{~min}$ ) after injection, where the contribution of efflux and metabolism can be neglected, was analyzed. Ignoring efflux, Eq. 2 integrates to as follows:

$$
C l_{\text {in }}=\frac{T(t)}{\int_{0}^{t} C(t) d t}=\frac{T(t)}{A U C_{0-t}}
$$

According to Eq. 1, the tissue uptake rate index was calculated using the amount of radioactivity in the tissue at the sampling time, and the area under the blood concentration time curve $(A U C)$ up to that time was obtained through curve fitting to the blood concentration time course based on a two-compartment model. Subsequently, the organ distribution clearance $\left(\mathrm{CL}_{\text {org }}\right)$ is expressed as follows:

$$
C L_{\text {org }}=C l_{\text {in }} \times W
$$

where $W(\mathrm{~g})$ is the total weight of the organ.

Preparation of Probucol Formulations for Pharmacological Study The probucol dose in the pharmacological study was set at $50 \mathrm{mg} / \mathrm{kg}$, because no cholesterol-lowering effect was observed at $20 \mathrm{mg} / \mathrm{kg}$ with HCO-60 micellar solution in the preliminary study. On the other hand, the lipid compositions of the injection formulations were the same as those of the distribution study, but their injection volumes were set at $10 \mathrm{ml} / \mathrm{kg}$. However, the solubilities of probucol were less than $2 \mathrm{mg} / \mathrm{ml}$ in the liposome and serum so that pharmacological study was performed only for the large emulsion, small emulsion, and HCO-60 micellar solution (Table 2). We did not examine the effect of the vehicles (lipid carriers) without drug, since none of the lipid carriers tested in this study have any such reported pharmacological activity. The oral administration of probucol solubilized in olive oil was examined at the same dose as a comparison.

Pharmacological Experiment of Probucol Male ddY mice $(22-25 \mathrm{~g})$ were fed cholesterol-rich foods containing $1.5 \%$ cholesterol, $0.5 \%$ sodium cholate, and $5 \%$ coconut oil for 7 days. A single administration of probucol formulations was done at a dose of $50 \mathrm{mg} / \mathrm{kg}$ by intravenous injection via the tail vein. Oral bolus administration was performed using a metallic gastric tube. At appropriate intervals $(0,12,18$, $24,30,36,42,48$, and $54 \mathrm{~h}$ ) after the administration, blood was collected from vena cava to obtain a plasma sample under ether anesthesia and the mice were sacrificed. The total plasma cholesterol concentration was measured by the cholesterol oxidase DAOS method ${ }^{17-19)}$ using Cholesterol E Test Wako (Wako Pure Chemicals Industry Ltd., Osaka). Statistical analysis was performed by Student's $t$-test.

Table 1. Formulation and Mean Diameters of Lipid Carrier Systems

\begin{tabular}{llcc}
\hline \multicolumn{1}{c}{ Lipid carrier systems } & \multicolumn{1}{c}{ Lipid composition } & Total lipid concentration (\%) & Mean diameter ${ }^{a}(\mathrm{~nm})$ \\
\hline HCO-60 micellar solution & Polyoxyethylene hydrogenated castor oil & 5 & $21.7 \pm 8.1$ \\
Liposome & Egg PC/egg SM (7:3) & 5 & $121.3 \pm 21.0$ \\
Large emulsion & Egg PC/soybean oil (0.12:1) & 5 & $277.6 \pm 67.0$ \\
Small emulsion with SM & Egg PC/egg SM/soybean oil $(0.7: 0.3: 1)$ & 5 & $102.4 \pm 20.0$ \\
\hline
\end{tabular}

a) Weight average distribution measured by dynamic laser scattering method is shown. 
Table 2. Formulations of Various Lipid Carrier Systems Employed for Probucol Administration

\begin{tabular}{llccc}
\hline \hline Lipid carrier systems & \multicolumn{1}{c}{ Composition } & $\begin{array}{c}\text { Probucol concentration } \\
(\mathrm{mg} / \mathrm{ml})\end{array}$ & $\begin{array}{c}\text { Total lipid }^{a)} \\
\text { concentration }(\%)^{(\%)}\end{array}$ & $\begin{array}{c}\text { Mean diameter } \\
(\mathrm{nm})\end{array}$ \\
\hline HCO-60 micellar solution & Polyoxyethylene hydrogenated castor oil & 5 & 5 & $19.0 \pm 5.7$ \\
Large emulsion & Egg PC/soybean oil (0.12:1) & 5 & 5 & $268.3 \pm 106.5$ \\
Small emulsion with SM & Egg PC/egg SM/soybean oil (0.7:0.3:1) & 5 & 5 & $83.9 \pm 39.8$ \\
\hline
\end{tabular}

a) Probucol content is included in total lipid concentration. b) Weight average distribution measured by dynamic laser scattering method is shown.
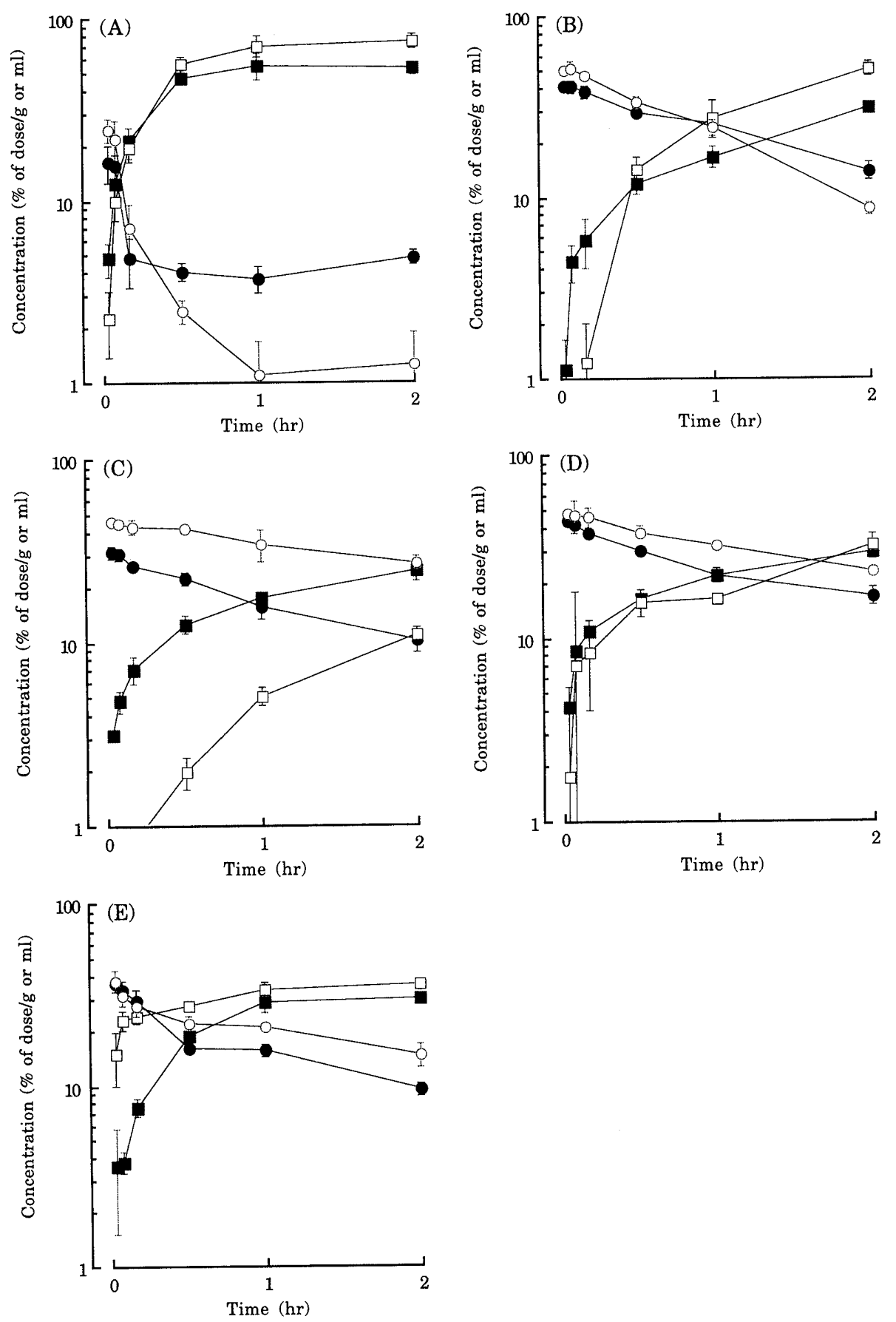

Fig. 1. Blood and Liver Concentration Profiles of $\left[{ }^{14} \mathrm{C}\right]$ Probucol and Lipid Carrier Particles Labeled with $\left[{ }^{3} \mathrm{H}\right]$ Cholesteryl Hexadecyl Ether after Intravenous Injection to Mice

(A) large emulsion; (B) small emulsion with SM; (C) HCO-60 micellar solution; (D) liposome; (E) mouse serum. $\bullet$, $\mathbf{m}$, probucol; $\bigcirc, \square$, lipid carrier particles. $\bigcirc$, $\bullet$, blood concentration; $\square, \boldsymbol{\square}$, liver concentration. Results are expressed as the mean \pm S.D. of at least three experiments. 


\section{RESULTS}

Lipophilicity of Probucol and Characteristics of Lipid Carrier Systems The log PCoct value, an index of lipophilicity, of probucol is calculated to be 10.75 , while that of cholesteryl hexadecyl ether (employed as a marker of lipid carriers) is 17.97. These calculated $\log \mathrm{PC}_{\text {oct }}$ values were employed as parameters of their lipophilicities, on the basis of their good correspondence $(r=0.96)$ to the experimentally obtained lipophilic indicies in reverse phase HPLC analysis. ${ }^{11)}$

The mean diameters of all lipid carriers are shown in Table 1. The incorporation of probucol resulted in little change in the diameters in a micellar solution, or in large or small emulsions (Table 2).

Blood Elimination and Liver Accumulation of Probucol Injected with Lipid Carriers The blood elimination and liver accumulation profiles of $\left[{ }^{14} \mathrm{C}\right]$ probucol and lipid carriers labeled by $\left[{ }^{3} \mathrm{H}\right]$ cholesteryl hexadecyl ether are shown in Fig. 1. The simultaneous determination of both compounds enabled the evaluation of their drug/carrier interactions in the blood circulation as well as the distribution profiles of each. Probucol was rapidly eliminated from blood circulation after intravenous injection with mouse serum, which is considered to represent the original distribution property of probucol, probably via lipoprotein transport. The in vivo fate of probucol was largely affected by injection with lipid carrier systems. By using a large emulsion, rapid elimination from the systemic circulation and accumulation in the liver, with more than $60 \%$ of dose at $1 \mathrm{~h}$ with emulsion particles, was observed. However, the probucol concentration in the liver decreased and the blood concentration was re-elevated at $1 \mathrm{~h}$ after administration. In contrast, a high blood concentration of probucol was retained in the cases of liposome and small emulsions corresponding to the long plasma circulation of the lipid carrier particles. That is, there was a relatively small difference in blood and liver concentration between probucol and lipid carriers in these formulations. In contrast, a large difference was observed in the blood concentration profiles between probucol and HCO-60 micelles, and this propensity was also the case with their concentration profiles in the liver.

Pharmacokinetic Parameters for Probucol Formulations The pharmacokinetic parameters for $\left[{ }^{14} \mathrm{C}\right]$ probucol
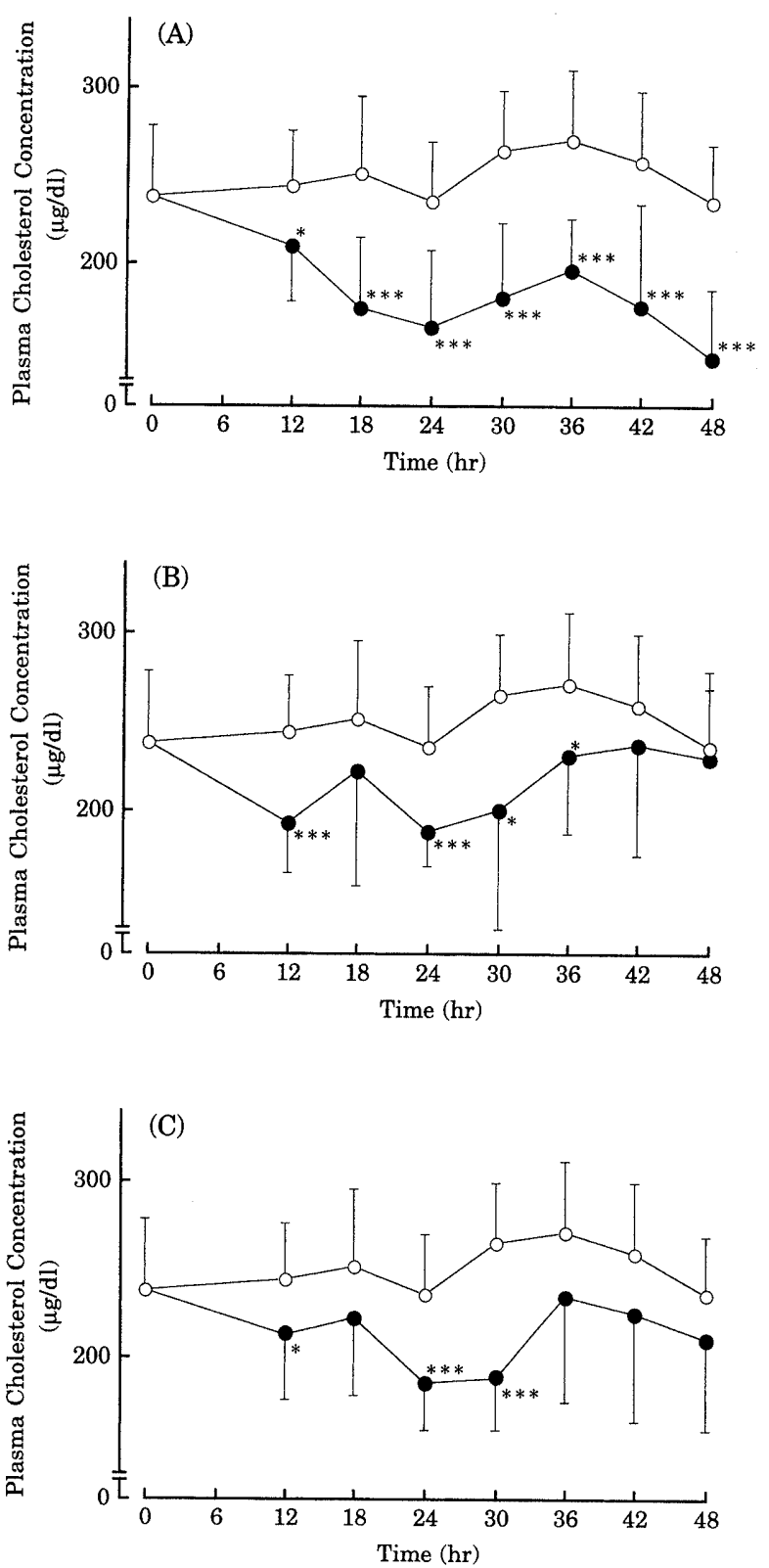

Fig. 2. Change in Total Plasma Cholesterol Concentration after Probucol Administration $(50 \mathrm{mg} / \mathrm{kg}$ ) with Various Formulations to Hyper-cholesterolemic Mice

(A) HCO-60 micellar solution; (B) large emulsion; (C) small emulsion with SM. $\mathrm{O}$, control; $\boldsymbol{O}$, probucol treatment. Results are expressed as the mean $\pm \mathrm{S}$. D. of at least ten experiments. Significantly different from that of control mice $(* p<0.05, * * * p<0.001)$ by the Student's $t$-test.

Table 3. Pharmacokinetic Parameters for Total Body Disposition of Model Lipophilic Drugs in the Early Phase after Intravenous Injection with Various Lipid Carrier Systems to Mice

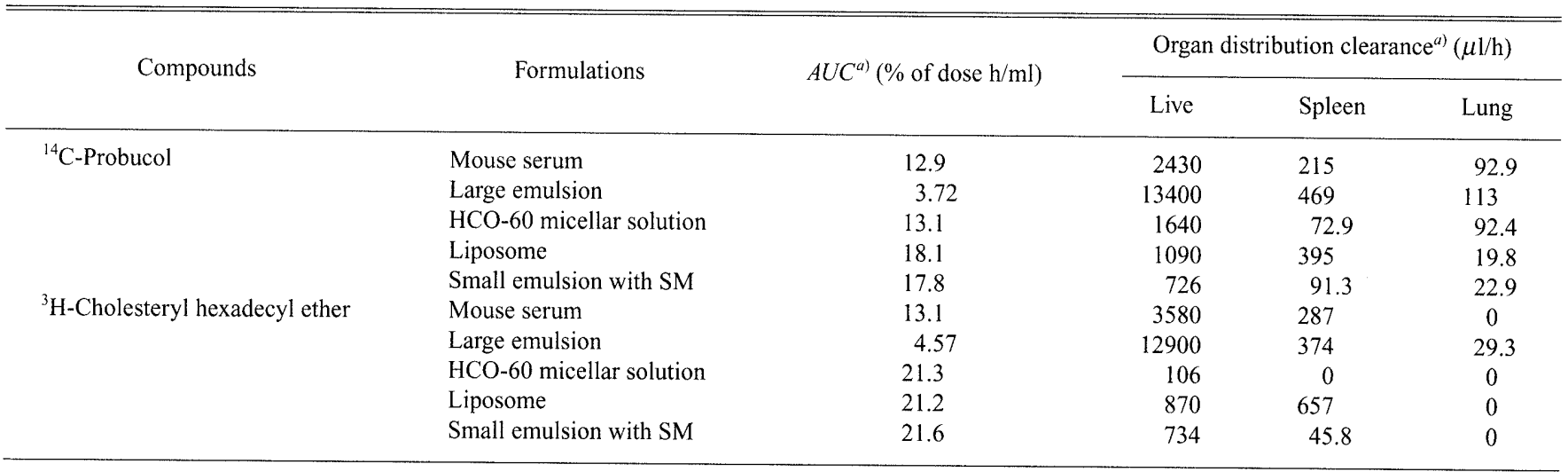

a) $A U C$ and organ distribution clearance were calculated for the initial phase of the experiment until 30 min after injection. 
Table 4. Plasma Cholesterol-Lowering Effects of Various Probucol Formulations

\begin{tabular}{lc}
\hline \hline \multicolumn{1}{c}{ Formulations } & $\begin{array}{c}\text { Integrated decrease of plasma cholesterol } \\
\text { concentration from control group }{ }^{a}(\mu \mathrm{g} \cdot \mathrm{h} / \mathrm{dl})\end{array}$ \\
\hline HCO-60 micellar solution & 3792 \\
Large emulsion & 1675 \\
Small emulsion with SM & 1721
\end{tabular}

a) Decreased area was calculated by numerical integration using a trapezoidal formula from the curves of treated and control group up to $48 \mathrm{~h}$ after administration.

and lipid particles are summarized in Table 3. To assess the distribution characteristics in the early phase with a minimal effect of efflux and metabolism, the parameters were calculated based on the data up to $30 \mathrm{~min}$. As for the parameter values of the carriers, long-circulating type carriers, i.e. liposome, small emulsion, and HCO-60 micellar solution, resulted in low organ clearances and consequently large $A U C$ values. In contrast, the large emulsion showed a large hepatic clearance. The $A U C$ and $C L$ values of probucol were not so different from those of carriers in all formulations except for the micellar solution. In the case of the micellar solution, a significantly large difference in the hepatic clearance between the micellar particles and probucol suggests its rapid release from micelles and subsequent distribution to the liver in a carrier-free form. The clearance values for the spleen and lung were relatively small.

Pharmacological Effect of Probucol Injected with Lipid Carrier Systems In hypercholesterolemia mice, the plasma cholesterol concentration was elevated and reached a level 3 -fold higher than in normal mice $2 \mathrm{~d}$ after the beginning of cholesterol-feeding, and then it was retained at the same level $(230-280 \mathrm{mg} / \mathrm{dl})$. There was interindividual variability in the plasma cholesterol level, since this was induced via free feeding. Preliminary study revealed that the cholesterol in the liver also increased to a level 3 times higher than that in the normal condition $3 \mathrm{~d}$ after feeding (data not shown). Thus, the hypercholesterolemic model mice were established $7 \mathrm{~d}$ after the beginning of feeding.

The plasma cholesterol-lowering effect of probucol was not observed in the case of a single oral administration at a dose of $50 \mathrm{mg} / \mathrm{kg}$ (data not shown). On the other hand, intravenous administration with lipid carrier systems showed a significant effect at the same dose, suggesting quite low bioavailability in oral administration. The peak time of the effect of probucol was $24 \mathrm{~h}$ after the injection in all dosage forms. The effect in the case of a micellar solution was larger than that of the emulsion formulations maintained for $48 \mathrm{~h}$ after injection. Although we did not test probucol in plasma due to its poor solubility, this formulation would not have shown any significant effect.

For comparing the cholesterol-lowering effects among various carrier systems, the area between the curves of the control group and each treatment group was calculated up to $48 \mathrm{~h}$ after administration, and the results are summarized in Table 4. In addition, the differences of effects between lipid carriers are also clear at $36 \mathrm{~h}$ after administration. That is, there is no significant difference between two types of emulsions, and the cholesterol level was restored to the control level $36 \mathrm{~h}$ after injection with each. In contrast, the micellar solution showed a significantly lower cholesterol level than all of the other formulations.

\section{DISCUSSION}

The application of lipid carrier systems with a solubilizing function is promising for the intravenous administration and specific delivery of highly lipophilic drugs. The interaction between lipophilic drugs and carrier particles must, however, be clarified to control the biodistribution of lipophilic drugs, taking into consideration the pharmacokinetic properties of lipid carriers. In our previous reports, ${ }^{10,11)}$ the biodistribution control of lipophilic drugs with lipid carrier systems was systematically studied and the lipophilicity of drugs and retention ability of carriers were demonstrated to be the major determinant factors. That is, the required lipophilicity of the drugs was clarified to be higher than $10^{6}$ for liposome, $10^{9}$ for emulsions and $10^{12}$ for non-ionic surfactant $\mathrm{HCO}-60$ micelles on the basis of the partition coefficient between $\mathrm{PC}_{\text {oct }}$. The present study was carried out to further elucidate a relationship between the delivery modality and pharmacological effect of lipophilic drugs using probucol as a model compound.

As shown in Fig. 1, the pharmacokinetic characteristics of probucol $\left(\mathrm{PC}_{\mathrm{oct}}=10^{10.8}\right)$ can be effectively controlled in all formulations, except in non-ionic surfactant $\mathrm{HCO}-60 \mathrm{mi}-$ celles in which probucol was released in the blood circulation and taken up by the liver in a free form. The uptake mode of probucol administered as a HCO-60 micelle formulation is considered to be advantageous for exerting its pharmacological effect. In contrast, the hepatic uptake with emulsion carrier particles resulted in a lower pharmacological effect, even though injection with small emulsion gave hepatic uptake of probucol apparently similar to that with micelles. The hepatic disposition of probucol taken up as emulsion particles differed from that of free drug. Our previous study ${ }^{12)}$ demonstrated that a large emulsion is preferentially taken up by the liver by non-parenchymal cells. On the other hand, small emulsion, liposome and $\mathrm{HCO}-60$ micelles are suggested to be slowly taken up by both the parenchymal and non-parenchymal liver cells. The differences in intrahepatic distribution are an important factor for pharmacological efficacy, since the parenchymal cells are the target of probucol. The small pharmacological action of a large emulsion would be due to its rapid sequestration by non-parenchymal cells in the liver. These results suggest the effects of the uptake mode by the liver on the pharmacological action of probucol.

In the design of lipophilic drug delivery with lipid carrier systems, the manner of distribution of drugs to the target organ plays an important role, as do other factors such as drug/carrier interaction and the biodistribution profile of the carrier.

\section{REFERENCES}

1) Yamamoto A., Matsuzawa Y., Kishino B., Hayashi R., Hirobe K., Kikkawa T., Atherosclerosis, 48, 157-166 (1983).

2) Collins-Gold L. C., Lyons R. T., Bartholow L. C., Adv. Drug Delivery Rev., 5, 189-208 (1990).

3) Davis S. S., Washington C., West P., Ann. N. Y. Acad. Sci., 507, 7588 (1987).

4) Juliano R. L., Adv. Drug Delivery Rev., 2, 31-54 (1988).

5) Kakutani T., Nishihara Y., Takahashi K., Hirano K., Proc. Int. Symp. 
Control. Rel. Bioact. Mater., 18, 359-360 (1991).

6) Mizushima Y., J. Bioact. Compatible Polymers, 3, 148-156 (1988).

7) Scherphof G. L., , "Targeted Drug Delivery," ed. by Juliano R. L., Springer-Verlag, Berlin, 1991, pp. 285-327.

8) Singh M., Ravin L. J., J. Pareter. Sci. Technol., 40, $34-41$ (1986).

9) Sakaeda (né Kakutani) T., Takahashi K., Nishihara Y., Hirano K., Biol. Pharm. Bull., 17, 1490-1495 (1997).

10) Takino T., Nakajima C., Takakura Y., Sezaki H., Hashida M., J. Drug Targeting, 1, 117-124 (1993).

11) Takino T., Konishi K., Takakura Y., Hashida M., Biol. Pharm. Bull., $17,121-125$ (1994).

12) Takino T., Nagahama E., Sakaeda (né Kakutani) T., Yamashita F.,
Takakura Y., Hashida M., Int. J. Pharmaceut., 114, 43-54 (1995).

13) Hansch C., Leo A., Unger S. H., Kim K. H., Nikaitani D., Lien E. J., J. Med. Chem., 16, 207-1216 (1973).

14) Leo A., Jow P. Y. C., Hansch C., J. Med. Chem., 18, 865-868 (1975).

15) Tokunaga Y., Iwasa T., Fujisaki J., Sawai S., Kagayama A., Chem. Pharm. Bull., 36, 3557-3564 (1988).

16) Takakura Y., Fujita T., Hashida M., Sezaki H., Pharm. Res., 7, 339 346 (1990).

17) Allain C. C., Poon L. S., Chan C. S. G., Richmond W., Fu P. C., Clin. Chem., 20, 470-475 (1974).

18) Richmond W., Scand. J. Clin. Lab. Invest., 29, 126 (1972).

19) Richmond W., Clin. Chem., 19, 1350-1356 (1973). 